\title{
ITERATIVE SOLUTION OF QUASILINEAR PARABOLIC EQUATIONS BY PARABOLIC EQUATIONS WITH CONSTANT COEFFICIENTS
}

\author{
JOHN E. LAVERY'
}

(Received 30 September 1980; revised 4 January 1982)

\begin{abstract}
A method for solving quasilinear parabolic equations of the types

$$
-\left[p\left(u_{x}, x, t\right)\right]_{x}+u_{t}=f, \quad-u_{x x}+p\left(u_{t}, x, t\right)=f
$$

that differs radically from previously known methods is proposed. For each initialboundary-value problem of one of these types that has boundary conditions of the first kind (second kind), a conjugate initial-boundary-value problem of the other type that has boundary conditions of the second kind (first kind) is defined. Based on the relations connecting the solutions of a pair of conjugate problems, a series of parabolic equations with constant coefficients that do not change step to step is constructed. The method proposed consists in calculating the solutions of the equations of this series. It is shown to have linear convergence. Results of a series of numerical experiments in a finite-difference setting show that one particular implementation of the proposed method has a smaller domain of convergence than Newton's method but that it sometimes converges faster within that domain.
\end{abstract}

\section{Introduction}

Numerical methods for solving quasilinear parabolic equations, which arise in heat flow with temperature-dependent coefficients and in other nonlinear diffusion processes, have been considered by Geymonat and Sibony [1], Hughes [2], Richtmyer and Morton [7], Samarskii [8] and many others. In the present article,

\footnotetext{
'Department of Business Mathematics, Soochow University, No. 1, Lane 129. Yen Ping South Road, Taipei, Taiwan 100, Republic of China; currently at Department of Mathematics, Case Western Reserve University, Cleveland, Ohio 44106, U.S.A.

(c) Copyright Australian Mathematical Society 1982
} 
we propose a method for solving quasilinear parabolic equations of the types

$$
\begin{gathered}
-\left[p\left(u_{x}, x, t\right)\right]_{x}+u_{t}=f(x, t), \\
-u_{x x}+p\left(u_{t}, x, t\right)=f(x, t),
\end{gathered}
$$

that differs radically from the methods found up to the present in the literature. For each parabolic initial-boundary-value problem of one of these types that has boundary conditions of the first kind (second kind), a conjugate problem of the other type that has boundary conditions of the second kind (first kind) is defined. From the relations connecting the solutions of a pair of conjugate problems, a series of parabolic equations with constant coefficients is constructed. The method proposed consists in calculating the solutions of the equations of this series. It can be used in conjunction with any convenient numerical scheme for solving linear parabolic equations. It is an adaption of a related method for solving quasilinear elliptic equations [5].

\section{Notation and assumptions}

We will consider equations (1.1) and (1.2) on the domain $D=(a, b) \times(0, T)$, where $a, b$ and $T$ are finite constants, $a<b$ and $T>0$. For the spaces to be used later on, we adopt notation similar to that of Ladyzhenskaya [4]. Let $W_{2,0}^{1}(a, b)$ denote $\left\{w \in W_{2}^{\prime}(a, b): w(a)=0, w(b)=0\right\}$, where $W_{2}^{\prime}(a, b)=\left\{w \in L_{2}(a, b)\right.$ : $\left.w^{\prime} \in L_{2}(a, b)\right\}$. Define $W_{2,0}^{1}(D)=\left\{u \in W_{2}^{1}(D): u(a, t)=0, u(b, t)=0\right.$ for almost all $t$ in $(0, T)\}$. Let $H^{0}(D)$ stand for $L_{2}(D)$ and $W_{2}^{1,0}(D)$ denote the Hilbert space $\left\{u \in H^{0}(D): u_{x} \in H^{0}(D)\right\}$ with inner product

$$
(u, v)_{W_{2}^{1,0}(D)}:=\iint_{D}\left(u v+u_{x} v_{x}\right) d D
$$

and corresponding norm. Define $V^{1,0}(D)$ to be the Banach space consisting of those elements $u$ of $W_{2}^{1,0}(D)$ for which $u(x, t)$ is in $L_{2}(a, b)$ for each $t, 0 \leqslant t \leqslant T$, and for which $\|u(x, t)\|_{L_{2}(a, b)}$ is a continuous function of $t$, with the norm

$$
\|u\|_{\nu^{1,0}(D)}:=\left[\left\|u_{x}\right\|_{H^{0}(D)}^{2}+\frac{1}{2} \max _{0<t<T}\|u(x, t)\|_{L_{2}(a, b)}^{2}\right]^{1 / 2} .
$$

Define $V_{0}^{1,0}(D)$ to be $\left\{u \in V^{1,0}(D): u(a, t)=0, u(b, t)=0\right.$ for almost all $t$ in $(0, T)\}$, a strict subspace of $V^{1,0}(D)$. Define $V^{2,1}(D)$ to be the Banach space of elements of $V^{1,0}(D)$ for which $u_{t}$ and $u_{x x}$ are in $H^{0}(D), u_{x}(x, t)$ is in $L_{2}(a, b)$ for 
each $t, 0 \leqslant t \leqslant T$, and $\left\|u_{x}(x, t)\right\|_{L_{2}(a, b)}$ is a continuous function of $t$, with the norm

$$
\begin{aligned}
& \|u\| \nu_{\nu^{2.1}(D)}:=\left[\left\|u_{x}\right\|_{H^{0}(D)}^{2}+\left\|u_{t}\right\|_{H^{0}(D)}^{2}+\left\|u_{x x}\right\|_{H^{0}(D)}^{2}+\frac{1}{2} \max _{0 \leqslant t \leqslant T}\|u(x, t)\|_{L_{2}(a, b)}^{2}\right. \\
& \left.+\max _{0 \leqslant t \leqslant T}\left\|u_{x}(x, t)\right\|_{L_{2}(a, b)}^{2}\right]^{1 / 2} .
\end{aligned}
$$

Let $V_{0}^{2,1}(D)=\left\{u \in V^{2,1}(D): u(a, t)=0, u(b, t)=0\right.$ for almost all $t$ in $\left.(0, T)\right\}$ and ${ }_{0} V^{2,1}(D)=\left\{u \in V^{2,1}(D): u_{x}(a, t)=0, u_{x}(b, t)=0\right.$ for almost all $t$ in $(0, T)\}$.

The function $p$ of equations (1.1) and (1.2) will be assumed to satisfy the following conditions (2.1):

For almost all fixed $(x, t)$ in $D, p(X, x, t) \in C^{1}\left(R^{1}\right)$.

There exist positive constants $m$ and $M$ such that,

for all $(X, x, t)$ in $R^{1} \times D, m \leqslant \frac{\partial p}{\partial X}(X, x, t) \leqslant M$.

For any function $X(x, t)$ in $H^{\circ}(D), p(X(x, t), x, t)$

is in $H^{0}(D)$; for any function $h(x, t)$ in $H^{0}(D)$,

the $X(x, t)$ defined uniquely (cf. condition (2.1b))

by $h(x, t)=p(X(x, t), x, t)$ is in $H^{0}(D)$.

In the conjugate problems defined in this paper, there will occur a function $\hat{p}$ that is related to $p$ as follows. Let $\alpha$ be any fixed element of $H^{0}(D)$ and $(x, t)$ be any fixed point of $D$. The transformation of $X \in R^{1}$ into $Y \in R^{1}$ defined by

$$
Y=p(X, x, t)+\alpha(x, t)
$$

is one-to-one and onto by condition (2.1b). Given any $\hat{\beta}$ in $H^{\circ}(D)$, one can define a function $\hat{p}$ by

$$
X=\hat{p}(Y, x, t)+\hat{\beta}(x, t) .
$$

This function $\hat{p}$ satisfies conditions (2.1) (with $\hat{m}=1 / M$ and $\hat{M}=1 / m$ instead of $m$ and $M$ in condition (2.1b)). For positive real numbers $\sigma$ and $\tau$, define $R(\sigma)$ and $\hat{R}(\tau)$ to be the smallest real numbers such that

$$
\begin{gathered}
\left|\frac{\partial p}{\partial X}(X, x, t)-\sigma\right| \leqslant R(\sigma), \\
\left|\frac{\partial \hat{p}}{\partial X}(X, x, t)-\tau\right| \leqslant \hat{R}(\tau)
\end{gathered}
$$


for all $(X, x, t)$ in $R^{1} \times D$. It will be assumed that there exist (positive) $\sigma$ and $\tau$ such that

$$
R(\sigma) \hat{R}(\tau)<1
$$

\section{Solution of quasilinear equations (I)}

It is our goal in this section to construct an iterative method for solving the first initial-boundary-value problem for equation (1.1) and the second initialboundary-value problem for equation (1.2). We first state these two problems precisely and develop relations that exist between their solutions. These relations then serve as the basis for constructing the method, which is shown to have linear convergence.

The first initial-boundary-value problem for equation (1.1) can be formulated as follows. Given $\alpha$ in $H^{0}(D)$ and $\beta$ in $V_{0}^{1,0}(D)$ such that

$$
f=\alpha_{x}-\beta_{t}
$$

and given $g$ in $L_{2}(a, b)$, find

$$
U \in V_{0}^{1.0}(D)
$$

such that

$$
\begin{gathered}
\int_{0}^{t} \int_{a}^{b}\left[p\left(U_{x}, x, t\right) h_{x}-U h_{t}\right] d x d t+\int_{a}^{b}[U(x, t)+\beta(x, t)] h(x, t) d x \\
=\int_{0}^{t} \int_{a}^{b}\left[-\alpha h_{x}+\beta h_{t}\right] d x d t+\int_{a}^{b}[g(x)+\beta(x, 0)] h(x, 0) d x
\end{gathered}
$$

for all $h$ in $W_{2,0}^{1}(D)$ and all $t$ in $(0, T]$. Problem (3.2) can be formally stated as the problem of finding $U$ such that

$$
\begin{gathered}
-\left[p\left(U_{x}, x, t\right)\right]_{x}+U_{t}=\alpha_{x}-\beta_{t} \equiv f \text { in } D, \\
U(x, 0)=g(x) \text { on }(a, b), \\
U(a, t)=0, \quad U(b, t)=0 \quad \text { on }(0, T) .
\end{gathered}
$$

(Equalities (3.3) and all subsequent equalities on $D,(a, b)$ and $(0, T)$ are understood to be "almost everywhere.") The initial condition (3.3b) is contained in equality (3.2b) and the boundary conditions (3.3c) are contained in condition (3.2a). With a slight change of notation, the second initial-boundary-value problem for equation (1.2) can be formulated as follows. Given $\hat{\alpha}$ in $V_{0}^{1,0}(D), \hat{\beta}$ in $H^{0}(D)$ and $\hat{g}$ in $W_{2}^{1}(a, b)$, find

$$
V \in_{0} V^{2,1}(D)
$$


such that

$$
\begin{gathered}
-V_{x x}+\hat{p}\left(V_{t}, x, t\right)=\hat{\alpha}_{x}-\hat{\beta} \text { in } D, \\
V(x, 0)=\hat{g}(x) \text { on }(a, b) .
\end{gathered}
$$

The $p$ and $f$ of equation (1.2) are replaced by $\hat{p}$ and $\hat{\alpha}_{x}-\hat{\beta}$ in equation (3.4b). The boundary conditions

$$
V_{x}(a, t)=0, \quad V_{x}(b, t)=0 \quad \text { on }(0, T)
$$

are contained in the space ${ }_{0} V^{2,1}(D)$ of condition (3.4a). We will assume that the data of problems (3.2) and (3.4) satisfy the relations

$$
\begin{gathered}
\hat{\alpha}=-\beta, \\
\hat{g}(x)=\int_{a}^{x}[g(\xi)+\beta(\xi, 0)] d \xi+c,
\end{gathered}
$$

where $c$ is an arbitrary constant of integration. A pair of problems (3.2) and (3.4), the data of which satisfy relations (2.1), (2.2) and (3.6) will be called conjugate problems.

The method for solving conjugate problems (3.2) and (3.4) that we will propose is based on relations that exist between the solutions of these problems. Assume that there exists a solution $U$ of problem (3.2). Define a function $V$ by the equalities

$$
\begin{gathered}
V_{x}=U+\beta, \\
V_{t}=p\left(U_{x}, x, t\right)+\alpha .
\end{gathered}
$$

The necessary and sufficient condition that such a $V$ exist is that $\left(V_{x}\right)_{t}=\left(V_{t}\right)_{x}$, that is, that

$$
[U+\beta]_{t}=\left[p\left(U_{x}, x, t\right)+\alpha\right]_{x},
$$

which holds since $U$ satisfies equality (3.2b) (and (3.3a)). Inverting relations (3.7), we have

$$
\begin{gathered}
U_{x}=\hat{p}\left(V_{t}, x, t\right)+\hat{\beta}, \\
U=V_{x}+\hat{\alpha}
\end{gathered}
$$

(cf. equalities (2.2) and (3.6a)). Equalities (3.8) imply that $V$ is a solution of the conjugate problem (3.4). Conversely, assuming that a solution $V$ of problem (3.4) exists, one can define a function $U$ by equality (3.8b). That equality (3.8a) holds for this $U$ is a consequence of equality (3.4b). This $U$ is, by equalities (3.7) (which follow from equalities (3.8)), a solution of problem (3.2). In summary, the solutions of problems (3.2) and (3.4), if they exist at all, occur in pairs that satisfy relations (3.7) and (3.8). 
Assume now that positive constants $\sigma$ and $\tau$ are given. (The roles of $\sigma$ and $\tau$ will be discussed below.) From equalities (3.7) and (3.8), it follows that $U$ and $V$ satisfy the following system of quasilinear parabolic problems:

$$
\begin{gathered}
V \in_{0} V^{2,1}(D), \\
-\sigma V_{x x}+V_{t}=-\sigma[U+\beta]_{x}+p\left(U_{x}, x, t\right)+\alpha \text { in } D, \\
V(x, 0)=\hat{g}(x) \quad \text { on }(a, b),
\end{gathered}
$$

and

$$
\begin{gathered}
U \in V_{0}^{1,0}(D) \\
\int_{0}^{t} \int_{a}^{b}\left[U_{x} h_{x}-\tau U h_{t}\right] d x d t+\tau \int_{a}^{b}[U(x, t)-\hat{\alpha}(x, t)] h(x, t) d x \\
=\int_{0}^{t} \int_{a}^{b}\left\{\left[\hat{p}\left(V_{t}, x, t\right)+\hat{\beta}-\tau V_{t}\right] h_{x}-\tau \hat{\alpha} h_{t}\right\} d x d t \\
+\tau \int_{a}^{b}[g(x)-\hat{\alpha}(x, 0)] h(x, 0) d x
\end{gathered}
$$

for all $h$ in $W_{2,0}^{1}(D)$ and all $t$ in $(0, T$ ]. For example, equality (3.9b) is obtained by differentiating equality (3.7a) with respect to $x$, multiplying it by $-\sigma$ and adding it to equality (3.7b).

Noting that any solution of problems (3.2) and (3.4) must satisfy equalities (3.7) and (3.8) and, hence, also equalities (3.9) and (3.10), we propose solving the quasilinear problems (3.2) and (3.4) by a "back door," that is, by solving system (3.9)-(3.10). We will do this by alternatively solving analogues of problems (3.9) and (3.10) in which the right-hand sides are known. Since the left-hand sides of equations (3.9b) and (3.10) are parabolic operators with constant coefficients, each iteration will consist of solving a relatively simple problem.

Let any element $u^{(n)}$ of $V_{0}^{1.0}(D)$ be given. Define $v^{(n)}$ to be the solution of the problem

$$
\begin{gathered}
v^{(n)} \in_{0} V^{2.1}(D), \\
-\sigma v_{x x}^{(n)}+v_{t}^{(n)}=-\sigma\left[u^{(n)}+\beta\right]_{x}+p\left(u_{x}^{(n)}, x, t\right)+\alpha \text { in } D, \\
v^{(n)}(x, 0)=\hat{g}(x) \quad \text { on }(a, b),
\end{gathered}
$$

(cf. problem (3.9)). Problem (3.11) is a well-posed linear parabolic problem with a unique solution (Ladyzhenskaya [4, page 178, Theorem 4.2]). On the other hand, given any element $v^{(n)}$ of ${ }_{0} V^{2,1}(D)$, a function $u^{(n+1)}$ can be found by solving the problem

$$
u^{(n+1)} \in V_{0}^{1,0}(D)
$$




$$
\begin{gathered}
\int_{0}^{t} \int_{a}^{b}\left[u_{x}^{(n+1)} h_{x}-\tau u^{(n+1)} h_{t}\right] d x d t+\tau \int_{a}^{b}\left[u^{(n+1)}(x, t)-\hat{\alpha}(x, t)\right] h(x, t) d x \\
=\int_{0}^{t} \int_{a}^{b}\left\{\left[\hat{p}\left(v_{t}^{(n)}, x, t\right)+\hat{\beta}-\tau v_{t}^{(n)}\right] h_{x}-\tau \hat{\alpha} h_{t}\right\} d x d t \\
\quad+\tau \int_{a}^{b}[g(x)-\hat{\alpha}(x, 0)] h(x, 0) d x
\end{gathered}
$$

for all $h$ in $W_{2,0}^{1}(D)$ and all $t$ in (0,T] (cf. problem (3.10)). Problem (3.12) is a well-posed linear parabolic problem with a unique solution (Ladyzhenskaya [4, page 171, Theorem 3.2]). Problem (3.12) can be formally restated as the problem of finding $u^{(n+1)}$ such that

$$
\begin{gathered}
-u_{x x}^{(n+1)}+\tau u_{t}^{(n+1)}=-\left[\hat{p}\left(v_{t}^{(n)}, x, t\right)+\hat{\beta}\right]_{x}+\tau\left[v_{x}^{(n)}+\hat{\alpha}\right]_{t} \text { in } D, \\
u^{(n+1)}(x, 0)=g(x) \text { on }(a, b), \\
u^{(n+1)}(a, t)=0, \quad u^{(n+1)}(b, t)=0 \text { on }(0, T) .
\end{gathered}
$$

The initial and boundary conditions (3.13b) and (3.13c) are contained in relations (3.12b) and (3.12a), respectively.

The method we wish to consider consists of iteratively solving problems (3.11) and (3.12), that is, of computing the sequence

$$
u^{(0)} \rightarrow v^{(0)} \rightarrow u^{(1)} \rightarrow v^{(1)} \rightarrow u^{(2)} \rightarrow \cdots,
$$

starting from any $u^{(0)}$ in $V_{0}^{1,0}(D)$, or the sequence

$$
v^{(0)} \rightarrow u^{(1)} \rightarrow v^{(1)} \rightarrow u^{(2)} \rightarrow v^{(2)} \rightarrow \cdots,
$$

starting from any $v^{(0)}$ in ${ }_{0} V^{2,1}(D)$. A formal proof of the convergence of the method will be presented below. We first explain on the basis of a linear example why convergence can be expected to occur at all and how one goes about choosing $\sigma$ and $\tau$.

Consider the model problem

$$
-d U_{x x}+U_{t}=\alpha_{x}-\beta_{t} \equiv f \text { in } D
$$

with initial and boundary conditions (3.3b) and (3.3c). Here, $d$ is a constant and $p(X, x, t)=d \cdot X$. With the $\hat{\alpha}$ of equality (3.6a), the $\hat{\beta}$ given by

$$
\hat{\beta}=-\alpha d^{-1}
$$

and the $\hat{g}$ of equality (3.6b), the conjugate problem is

$$
-V_{x x}+d^{-1} V_{t}=\hat{\alpha}_{x}-\hat{\beta} \text { in } D
$$

with initial and boundary conditions (3.4c) and (3.5). Relations (3.7) and (3.8) are, for this pair of conjugate problems,

$$
\begin{gathered}
V_{x}=U+\beta, \quad V_{t}=d \cdot U_{x}+\alpha, \\
U_{x}=d^{-1} \cdot V_{t}+\hat{\beta}, \quad U=V_{x}+\hat{\alpha} .
\end{gathered}
$$


The linear equations (3.11b) and (3.13a) that are to be used to solve the model "quasilinear" equations (3.15) and (3.17) are

$$
\begin{gathered}
-\sigma v_{x x}^{(n)}+v_{t}^{(n)}=(d-\sigma) u_{x}^{(n)}-\sigma \beta_{x}+\alpha \text { in } D, \\
-u_{x x}^{(n+1)}+\tau u_{t}^{(n+1)}=\left(\tau-d^{-1}\right) v_{x t}^{(n)}+\tau \hat{\alpha}_{t}-\hat{\beta}_{x} \text { in } D .
\end{gathered}
$$

In the right-hand side of equation (3.20), we note that the positive constants $d$ and $\sigma$ in the coefficient of $u_{x}^{(n)}$ cancel each other, at least partially. In the right-hand side of equation (3.21), $\tau$ and $d^{-1}$ in the coefficient of $v_{x t}^{(n)}$ also (partially) cancel each other out. This situation raises the expectation that, if the amount of cancellation is sufficiently large, convergence will occur. This is indeed the case, as we will see in the theorem below. In order to guarantee linear convergence, $\sigma$ and $\tau$ must merely be chosen so that the ratio of convergence $R(\sigma) \hat{R}(\tau)$, which, for the present example, equals $|d-\sigma| \cdot\left|d^{-1}-\tau\right|$, is less than unity. The optimum $\sigma$ and $\tau$ are easily found. If we choose $\sigma=d$ and $\tau=d^{-1}$, convergence occurs on the first iteration, since the term involving $u_{x}^{(n)}$ in equation (3.20) and the term involving $v_{x t}^{(n)}$ in equation (3.21) disappear. In this case, the linear equations (3.20) and (3.21) of the proposed method coincide with the model "quasilinear" equations (3.17) and (3.15), respectively.

This realization of how the choice of $\sigma$ and $\tau$ controls convergence of the method for the model equation (3.15) can easily be extended to the original quasilinear problem (3.2). The rate of convergence depends on how much the terms $\sigma u_{x}^{(n)}$ and $p\left(u_{x}^{(n)}, x, t\right)$ on the right-hand side of equation $(3.11 \mathrm{~b})$ cancel each other out and how much the terms $\left[\hat{p}\left(v_{t}^{(n)}, x, t\right)\right]_{x}$ and $\tau\left[v_{x}^{(n)}\right]_{t}$ on the right-hand side of equation (3.13a) cancel each other out. Roughly speaking, the greater the cancellation is, the smaller the quantity $R(\sigma) \hat{R}(\tau)$ will be. Linear convergence is guaranteed whenever this quantity, the ratio of convergence, is less than unity. A precise rule for choosing the optimum $\sigma$ and $\tau$ has not been determined. However, on the basis of the example above and the numerical results presented below in Section 5, the author expects that $\sigma$ should be some kind of weighted average of $\partial p(X, x, t) / \partial X$ and $\tau$ should be $1 / \sigma$.

To close this section, we present a formal statement and proof of the convergence of the method.

Theorem 1. Let condition (2.4) be fulfilled. Then the conjugate problems (3.2) and (3.4) have unique solutions $U$ and $V$. The pair $\{U, V\}$ is the unique set of solutions of the system (3.9)-(3.10). The iterates $u^{(n)}$ and $v^{(n)}$ of sequences (3.14) converge linearly in $V_{0}^{1,0}(D)$ to $U$ and linearly in ${ }_{0} V^{2,1}(D)$ to $V$, respectively. The a priori rate of convergence is in both cases $R(\sigma) \hat{R}(\tau)$. 
Proor. Replace $n$ by $n-1$ in equality (3.11b), subtract that equality from equality $(3.11 \mathrm{~b})$ as it stands, multiply the resulting equality by $v^{(n)}-v^{(n-1)}$ and integrate over $(a, b) \times(0, t)$. Making use of the boundary conditions for $v^{(n)}$ and $v^{(n-1)}$ contained in the space ${ }_{0} V^{2,1}(D)$, of the initial condition (3.11c) and of an intermediate-value theorem, we obtain

$$
\begin{aligned}
\sigma \int_{0}^{t} \int_{a}^{b}\left(v_{x}^{(n)}-v_{x}^{(n-1)}\right)^{2} d x d t+\frac{1}{2} \int_{a}^{b}\left[v^{(n)}(x, t)-v^{(n-1)}(x, t)\right]^{2} d x \\
\quad=\int_{0}^{t} \int_{a}^{b}\left[\frac{\partial p}{\partial\left(u_{x}\right)}\left(u_{x}, x, t\right)-\sigma\right]\left(u_{x}^{(n)}-u_{x}^{(n-1)}\right)\left(v^{(n)}-v^{(n-1)}\right) d x d t,
\end{aligned}
$$

for all $t$ in $(0, T]$, where $u=u^{(n-1)}+c\left(u^{(n)}-u^{(n-1)}\right)$ for some constant $c$, $0<c<1$. Letting $D_{t}$ denote $(a, b) \times(0, t)$ and recalling inequality (2.3a), one obtains from equality (3.22) the inequality

$$
\begin{aligned}
\sigma\left\|v_{x}^{(n)}-v_{x}^{(n-1)}\right\|_{H^{0}\left(D_{t}\right)}^{2}+\frac{1}{2}\left\|v^{(n)}(x, t)-v^{(n-1)}(x, t)\right\|_{L_{2}(a, b)}^{2} \\
\quad \leqslant R(\sigma)\left\|u_{x}^{(n)}-u_{x}^{(n-1)}\right\|_{H^{0}\left(D_{f}\right)}\left\|v^{(n)}-v^{(n-1)}\right\|_{H^{0}\left(D_{t}\right)} .
\end{aligned}
$$

Analogously, one can obtain

$$
\begin{gathered}
\left\|v_{t}^{(n)}-v_{t}^{(n-1)}\right\|_{H^{0}\left(D_{t}\right)}^{2}+(\sigma / 2)\left\|v_{x}^{(n)}(x, t)-v_{x}^{(n-1)}(x, t)\right\|_{L_{2}(a, b)}^{2} \\
\leqslant R(\sigma)\left\|u_{x}^{(n)}-u_{x}^{(n-1)}\right\|_{H^{0}\left(D_{t}\right)}\left\|v_{t}^{(n)}-v_{t}^{(n-1)}\right\|_{H^{0}\left(D_{t}\right)} \\
\sigma\left\|v_{x x}^{(n)}-v_{x x}^{(n-1)}\right\|_{H^{0}\left(D_{t}\right)}^{2}+\frac{1}{2}\left\|v_{x}^{(n)}(x, t)-v_{x}^{(n-1)}(x, t)\right\|_{L_{2}(a, b)}^{2} \\
\quad \leqslant R(\sigma)\left\|u_{x}^{(n)}-u_{x}^{(n-1)}\right\|_{H^{0}\left(D_{t}\right)}\left\|v_{x x}^{(n)}-v_{x x}^{(n-1)}\right\|_{H^{0}\left(D_{l}\right)}
\end{gathered}
$$

for all $t$ in $(0, T]$. Replacing $t$ by $T$ on the right sides of inequalities (3.23), taking the maxima of the left sides, adding the resulting inequalities and simplifying yields

$$
\left\|v^{(n)}-v^{(n-1)}\right\| v^{2,1}(D) \leqslant r R(\sigma)\left\|u_{x}^{(n)}-u_{x}^{(n-1)}\right\|_{H^{0}(D)},
$$

where

$$
r=\frac{\sqrt{3} \max (1, \sqrt{2 T})}{\min (1, \sigma)} .
$$

From inequality (3.23b), one can also derive the inequality

$$
\left\|v_{t}^{(n)}-v_{t}^{(n-1)}\right\|_{H^{0}(D)} \leqslant R(\sigma)\left\|u_{x}^{(n)}-u_{x}^{(n-1)}\right\|_{H^{0}(D)} .
$$

By similar reasoning, one can show that

$$
\left\|u^{(n+1)}-u^{(n)}\right\| \mathcal{V}^{1.0}(D) \leqslant \frac{\hat{R}(\tau)}{\min (1, \tau)}\left\|v_{t}^{(n)}-v_{t}^{(n-1)}\right\|_{H^{0}(D)},
$$




$$
\left\|u_{x}^{(n+1)}-u_{x}^{(n)}\right\|_{H^{0}(D)} \leqslant \hat{R}(\tau)\left\|v_{t}^{(n)}-v_{t}^{(n-1)}\right\|_{H^{0}(D)} .
$$

Inequalities (3.25) and (3.27) imply that

$$
\begin{aligned}
& \left\|u_{x}^{(n+1)}-u_{x}^{(n)}\right\|_{H^{0}(D)} \leqslant R(\sigma) \hat{R}(\tau)\left\|u_{x}^{(n)}-u_{x}^{(n-1)}\right\|_{H^{0}(D)}, \\
& \left\|v_{t}^{(n+1)}-v_{t}^{(n)}\right\|_{H^{0}(D)} \leqslant R(\sigma) \hat{R}(\tau)\left\|v_{t}^{(n)}-v_{t}^{(n-1)}\right\|_{H^{0}(D)} .
\end{aligned}
$$

Inequalities (2.4), (3.26) and (3.29) imply that, for any $n \geqslant 2$ and any $k \geqslant 1$,

$$
\begin{aligned}
\left\|u^{(n+k)}-u^{(n)}\right\|_{\mathcal{V}^{1.0}(D)} & \leqslant \sum_{i=n}^{n+k-1}\left\|u^{(t+1)}-u^{(l)}\right\|_{v^{1,0}(D)} \\
& \leqslant \frac{\hat{R}(\tau)}{\min (1, \tau)} \sum_{i=n}^{n+k-1}\left\|v_{t}^{(l)}-v_{t}^{(i-1)}\right\|_{H^{0}(D)} \\
& \leqslant \frac{\hat{R}(\tau)}{\min (1, \tau)} \sum_{i=n}^{n+k-1}[R(\sigma) \hat{R}]^{i-2}\left\|v_{t}^{(2)}-v_{t}^{(1)}\right\|_{H^{0}(D)} \\
& \leqslant \frac{R(\sigma)^{n-2} \hat{R}(\tau)^{n-1}}{[1-R(\sigma) \hat{R}(\tau)] \min (1, \tau)}\left\|v_{t}^{(2)}-v_{t}^{(1)}\right\|_{H^{0}(D)} .
\end{aligned}
$$

Analogously, inequalities (2.4), (3.24) and (3.28) imply that, for any $n \geqslant 2$ and any $k \geqslant 1$,

$$
\left\|v^{(n+k)}-v^{(n)}\right\| v^{2.1 .(D)} \leqslant \frac{r R(\sigma)^{n} \hat{R}(\tau)^{n-1}}{1-R(\sigma) \hat{R}(\tau)}\left\|u_{x}^{(2)}-u_{x}^{(1)}\right\|_{H^{0}(D)} .
$$

Inequalities (3.30) and (3.31) are statements that $\left\{u^{(n)}\right\}$ and $\left\{v^{(n)}\right\}$ are Cauchy sequences in $V_{0}^{1,0}(D)$ and ${ }_{0} V^{2,1}(D)$, respectively. Define $U=\lim _{n \rightarrow \infty} u^{(n)}$ and $V=\lim _{n \rightarrow \infty} v^{(n)}$. Taking the limit in equality $(3.12 \mathrm{~b})$, we obtain equality $(3.10 \mathrm{~b})$. Taking the limit in equalities (3.11b) and (3.11c), we obtain equalities (3.9b) and (3.9c). Choosing $h=U+\beta-V_{x}$ in equality (3.10b) and using the equality

$$
U_{x}=\hat{p}\left(V_{t}+\sigma\left(U_{x}+\beta_{x}-V_{x x}\right), x, t\right)+\hat{\beta},
$$

which is a consequence of equality (3.9b) and relations (2.2), one can show that equality (3.7a) holds. Even if $h=U+\beta-V_{x}$ is not as smooth as required for equality (3.10b), equality (3.7a) still holds and can be proved by a limiting argument. Equalities (3.7a) and (3.9b) imply that equality (3.7b) holds. Since equalities (3.7) hold, equalities (3.8) also hold. It follows from relations (3.6), (3.7) and (3.8) that any solutions $U$ and $V$ of problems (3.9) and (3.10) are solutions of problems (3.2) and (3.4) and vice versa. That the solutions of these problems are unique and that the rates of convergence of $u^{(n)}$ to $U$ and of $v^{(n)}$ to $V$ are linear with ratio $R(\sigma) \hat{R}(\tau)$ can be shown using inequalities similar to inequalities (3.28) and (3.29). The theorem is proved. 
We note in passing that arguments similar to those by which inequalities (3.30) and (3.31) were derived can be used to obtain the following a posteriori error bounds:

$$
\begin{aligned}
& \left\|u^{(n)}-U\right\|_{V^{1,0}(D)} \leqslant \frac{R(\sigma) \hat{R}(\tau)}{[1-R(\sigma) \hat{R}(\tau)] \min (1, \tau)}\left\|u_{x}^{(n)}-u_{x}^{(n-1)}\right\|_{H^{0}(D)}, \\
& \left\|v^{(n)}-V\right\| v^{2,1}(D) \leqslant \frac{r R(\sigma) \hat{R}(\tau)}{1-R(\sigma) \hat{R}(\tau)}\left\|v_{t}^{(n)}-v_{t}^{(n-1)}\right\|_{H^{0}(D)} .
\end{aligned}
$$

\section{Solution of quasilinear equations (II)}

We now consider solving the first initial-boundary-value problem for equation (1.2) in $V_{0}^{2,1}(D)$ and solving the second initial-boundary-value problem for equation $(1.1)$ in $V^{1,0}(D)$. Since the results of this section are analogous to those of Section 3, comments and explanation will here be held to a minimum.

The two problems to be considered can be formulated as follows. Given $\alpha$ in $H^{0}(D)$ and $\beta$ in $V^{1,0}(D)$ such that $f=\beta_{x}-\alpha$ and given $g$ in $W_{2,0}^{1}(a, b)$, find

$$
U \in V_{0}^{2,1}(D)
$$

such that

$$
\begin{gathered}
-U_{x x}+p\left(U_{t}, x, t\right)=\beta_{x}-\alpha \equiv f \text { in } D, \\
U(x, 0)=g(x) \text { on }(a, b) .
\end{gathered}
$$

With a slight change of notation, the second of these problems can be stated as follows. Given $\hat{\alpha}$ in $V^{1,0}(D)$ and $\hat{\beta}$ in $H^{0}(D)$ and given $\hat{g}$ in $L_{2}(a, b)$, find

$$
V \in V^{1,0}(D)
$$

such that

$$
\begin{gathered}
\int_{0}^{t} \int_{a}^{b}\left[\hat{p}\left(V_{x}, x, t\right) h_{x}-V h_{t}\right] d x d t+\int_{a}^{b}[V(x, t)+\hat{\alpha}(x, t)] h(x, t) d x \\
=\int_{0}^{t} \int_{a}^{b}\left(-\hat{\beta} h_{x}+\hat{\alpha} h_{t}\right) d x d t+\int_{a}^{b}[\hat{g}(x)+\hat{\alpha}(x, 0)] h(x, 0) d x
\end{gathered}
$$

for all $h$ in $W_{2}^{1}(D)$ and all $t$ in $(0, T]$. The $p$ and $f$ of equation (1.1) are replaced by $\hat{p}$ and $\hat{\beta}_{x}-\hat{\alpha}_{t}$ to create equation (4.2). We will assume that

$$
\hat{\alpha}=-\beta, \quad \hat{g}(x)=g^{\prime}(x)+\beta(x, 0) .
$$


A pair of problems (4.1) and (4.2) for which conditions (2.1), (2.2) and (4.3) hold will be called a pair of conjugate problems. The relations

$$
\begin{aligned}
& V_{x}=p\left(U_{t}, x, t\right)+\alpha, \quad V=U_{x}+\beta, \\
& U_{x}=V+\hat{\alpha}, \quad U_{t}=\hat{p}\left(V_{x}, x, t\right)+\hat{\beta},
\end{aligned}
$$

hold between the solutions of problem (4.1) and (4.2) (cf. relations (3.7) and (3.8)).

We propose the following method for solving a pair of conjugatc problems. Let positive constants $\sigma$ and $\tau$ be given. Let $u^{(n)}$ be any element of $V_{0}^{2,1}(D)$. The problem of finding

$$
v^{(n)} \in V^{1,0}(D)
$$

such that

$$
\begin{aligned}
\int_{0}^{t} \int_{a}^{b}\left(v_{x}^{(n)} h_{x}-\right. & \left.\sigma v^{(n)} h_{t}\right) d x d t+\sigma \int_{a}^{b}\left[v^{(n)}(x, t)-\beta(x, t)\right] h(x, t) d x \\
= & \int_{0}^{t} \int_{a}^{b}\left\{\left[p\left(u_{t}^{(n)}, x, t\right)+\alpha-\sigma u_{t}^{(n)}\right] h_{x}-\sigma \beta h_{t}\right\} d x d t \\
& +\sigma \int_{a}^{b}[\hat{g}(x)-\beta(x, 0)] h(x, 0) d x
\end{aligned}
$$

for all $h$ in $W_{2}^{1}(D)$ and all $t$ in $(0, T]$ is a well-posed parabolic problem with a unique solution (Ladyzhenskaya [4, page 178, Theorem 4.1 and page 180, Remark]). Let $v^{(n)}$ be any element of $V^{1,0}(D)$. The problem of finding

$$
u^{(n+1)} \in V_{0}^{2,1}(D)
$$

such that

$$
\begin{gathered}
-\tau u_{x x}^{(n+1)}+u_{t}^{(n+1)}=-\tau\left[v^{(n)}+\hat{\alpha}\right]_{x}+\hat{p}\left(v_{x}^{(n)}, x, t\right)+\hat{\beta} \text { in } D, \\
u^{(n+1)}(x, 0)=g(x) \text { on }(a, b)
\end{gathered}
$$

is a well-posed parabolic problem with a unique solution (Ladyzhenskaya [4, page 158, Theorem 2.1]). Problems (4.6) and (4.7) are obtained from relations (4.4) and (4.5) in the same way that problems (3.11) and (3.12) were derived from relations (3.7) and (3.8). The method we propose consists of iteratively solving problems (4.6) and (4.7), that is, computing sequence (3.14a) starting from any $u^{(0)}$ in $V_{0}^{2,1}(D)$ or sequence $(3.14 \mathrm{~b})$ starting from any $v^{(0)}$ in $V^{1,0}(D)$. The comments in Section 3 concerning why convergence can be expected to occur apply with minor changes here. It is expected that $\sigma$ should be chosen to be some kind of weighted average of $\partial p(X, x, t) / \partial X$ and that $\tau$ should be $1 / \sigma$. The formal statement of the convergence of the method is contained in the following theorem. 
THEOREM 2. Let condition (2.4) be fulfilled. Then the conjugate problems (4.1) and (4.2) have unique solutions $U$ and $V$. The iterates $u^{(n)}$ and $v^{(n)}$ of sequences (3.14) (obtained by iteratively solving problems (4.6) and (4.7) instead of problems (3.11) and (3.12)) converge linearly in $V_{0}^{2,1}(D)$ to $U$ and linearly in $V^{1,0}(D)$ to $V$, respectively. The a priori rate of convergence is in both cases $R(\sigma) \hat{R}(\tau)$.

The proof of Theorem 2 is similar to that of Theorem 1 and is omitted. $A$ posteriori error bounds analogous to inequalities (3.32) hold:

$$
\left\|u^{(n)}-U\right\|_{v^{2,1}(D)} \leqslant \frac{\hat{r} R(\sigma) \hat{R}(\tau)}{1-R(\sigma) \hat{R}(\tau)}\left\|u_{t}^{(n)}-u_{t}^{(n-1)}\right\|_{H^{0}(D)},
$$

where

$$
\hat{r}=\frac{\sqrt{3}}{\min (1, \tau)} \max \left\{1,\left[\frac{2 T(b-a)^{2}}{2 \pi^{2}(T+1)+(b-a)^{2}}\right]^{1 / 2}\right\},
$$

and

$$
\left\|v^{(n)}-V\right\|_{V^{1,0}(D)} \leqslant \frac{R(\sigma) \hat{R}(\tau)}{[1-R(\sigma) \hat{R}(\tau)] \min (1, \sigma)}\left\|v_{x}^{(n)}-v_{x}^{(n-1)}\right\|_{H^{0}(D)} .
$$

\section{Numerical implementation and comparison with other methods}

The above discussion is not based on any one numerical procedure but is carried out on the level of the differential equation before discretization. This fact is of considerable importance, since it implies that the proposed method can be used with any numerical procedure, such as finite differences or finite elements, appropriate for solving linear parabolic equations. We will describe here one way of numerically implementing the method proposed in Section 3 and compare it with solution of quasilinear parabolic equations by the commonly used Newton's method. Before proceeding to the description of the implementations of these methods, we make the following observation.

For the global convergence discussed in Sections 3 and 4, the functions $p$ and $\hat{p}$ are required to satisfy conditions (2.3) and (2.4). These are rather strict requirements that will in practice often not be satisfied globally. If they are, however, satisfied locally for the argument $X$ of $p(X, x, t)$ and the argument $Y$ of $\hat{p}(Y, x, t)$ in some fixed intervals, the method proposed in this paper will converge as long as the arguments $u_{x}^{(n)}$ or $u_{t}^{(n)}$ of $p$ and $v_{t}^{(n)}$ or $v_{x}^{(n)}$ of $\hat{p}$ or the numerical approximations of these arguments remain within the fixed intervals. The purpose of the numerical experiments, the results of which are presented later in this section, is to investigate local convergence for the quasilinear problem (3.3). 
To solve problem (3.3) by finite differences, we will use uniform meshes with length $\Delta x=(b-a) / I$ in the $x$-direction and $\Delta t=T / J$ in the $t$-direction, where $I$ and $J$ are integers $\geqslant 2$. The mesh points will be $\left(x_{i}, t_{j}\right)=(a+i \Delta x, j \Delta t)$, where $i$ is an integer between 0 and $I$ (for $U$ ) or an integer plus one-half between $-1 / 2$ and $I+1 / 2$ (for $V$ ) and $j$ is an integer between 0 and $J$. The finite-difference approximation of any unknown function and the value of any known function at $\left(x_{t}, t_{j}\right)$ will be denoted by adding a subscript $i$ and a superscript $j$. For example, $u_{i}^{(n) j}$ and $V_{i}^{j}$ are the approximations of $u^{(n)}\left(x_{i}, t_{j}\right)$ and $V\left(x_{i}, t_{j}\right)$ and $f_{i}^{j}$ stands for $f\left(x_{l}, t_{j}\right)$. All finite-difference equations will be based on the implicit " $T$ " scheme with truncation error $O\left((\Delta x)^{2}+\Delta t\right)$.

We describe first the implementation of Newton's method (cf. Richtmyer and Morton [7, Section 8.6]). Equations (3.3a) and (3.3c) are approximated by the nonlinear system of equations

$$
\begin{gathered}
-\frac{1}{\Delta x}\left[p\left(\frac{U_{i+1}^{j+1}-U_{i}^{j+1}}{\Delta x}, x_{i+1 / 2}, t_{j+1}\right)-p\left(\frac{U_{i}^{j+1}-U_{i-1}^{j+1}}{\Delta x}, x_{i-1 / 2}, t_{j+1}\right)\right] \\
+\frac{U_{l}^{j+1}-U_{i}^{J}}{\Delta t}=f_{i}^{j+1 / 2}, \quad i=1, \ldots, I-1 \\
U_{0}^{j+1}=U_{I}^{j+1}=0
\end{gathered}
$$

In what follows, $j$ is considered to be a fixed integer, $0 \leqslant j \leqslant J-1$. When solving system (5.1) by Newton's method, one calculates the $(n+1)$-st approximations $u_{i}^{(n+1) j+1}$ of the $U_{i}^{j+1}$ from the $n$th approximations $u_{i}^{(n) j+1}$ and the known values $U_{i}^{J}$ of the previous time step by solving the linear system

$$
\begin{gathered}
-\frac{1}{\Delta x}\left[p^{\prime}\left(\frac{u_{i+1}^{(n) j+1}-u_{i}^{(n) j+1}}{\Delta x}, x_{i+1 / 2}, t_{j+1}\right)\left(\frac{w_{i+1}-w_{i}}{\Delta x}\right)\right. \\
\left.-p^{\prime}\left(\frac{u_{i}^{(n) j+1}-u_{i-1}^{(n) j+1}}{\Delta x}, x_{i-1 / 2}, t_{j+1}\right)\left(\frac{w_{i}-w_{i-1}}{\Delta x}\right)\right]+\frac{w_{i}}{\Delta t} \\
=f_{i}^{j+1 / 2}+\frac{1}{\Delta x}\left[p\left(\frac{u_{i+1}^{(n) j+1}-u_{i}^{(n) j+1}}{\Delta x}, x_{i+1 / 2}, t_{j+1}\right)\right. \\
-\frac{u_{i}^{(n) j+1}-U_{i}^{j}}{\Delta t}, \quad i=1, \ldots, I-1, \\
w_{0}=w_{l}=0
\end{gathered}
$$

for the $w_{t}$ and setting

$$
u_{i}^{(n+1) j+1}=u_{i}^{(n) j+1}+w_{i}, \quad i=1, \ldots, I-1 .
$$


For each given $j$, one iteratively solves system (5.2) starting from the initial approximations $u_{i}^{(0) j+1}=U_{i}^{J}$ (on the first time step, one uses the initial condition (3.3b) in the form $U_{i}^{0}=g\left(x_{i}\right)$ ) and accepts $u_{i}^{(n+1) j+1}$ as $U_{t}^{j+1}$ for the first $n$ such that

$$
\max _{1 \leqslant i \leqslant I-1}\left|u_{i}^{(n+1) j+1}-u_{i}^{(n) j+1}\right| \leqslant \varepsilon_{1} .
$$

One then proceeds to repeat the process again on the next time step.

The implementation of the method proposed in the present paper is as follows. Equation (3.11b) is approximated by

$$
\begin{aligned}
-\sigma \frac{v_{l+1 / 2}^{(n) j+1}-2 v_{i-1 / 2}^{(n) j+1}+v_{i-3 / 2}^{(n)}+1}{(\Delta x)^{2}}+\frac{v_{i-1 / 2}^{(n) j+1}-V_{i-1 / 2}^{J}}{\Delta t} \\
=-\sigma \frac{u_{i}^{(n) j+1}+U_{i}^{J}+\beta_{i}^{j+1 / 2}-u_{i-1}^{(n) j+1}-U_{i-1}^{j}-\beta_{i-1}^{++1 / 2}}{2 \Delta x} \\
+p\left(\frac{u_{i}^{(n) j+1}-u_{i-1}^{(n) j+1}+U_{i}^{j}-U_{i-1}^{j}}{2 \Delta x}, x_{i-1 / 2}, t_{j+1 / 2}\right) \\
+\alpha_{i-1 / 2}^{j+1 / 2}, \quad i=1, \ldots, I .
\end{aligned}
$$

The homogeneous Neumann boundary conditions contained in the space ${ }_{0} V^{2,1}(D)$ of condition (3.11a) are approximated to second order by

$$
v_{-1 / 2}^{(n) J+1}=v_{1 / 2}^{(n) j+1}, \quad v_{I+1 / 2}^{(n)+1}=v_{I-1 / 2}^{(n) j+1} .
$$

Equations (3.13a) and (3.13c) are approximated by

$$
\begin{aligned}
& -\frac{u_{i+1}^{(n+1) j+1}-2 u_{i}^{(n+1) j+1}+u_{i-1}^{(n+1) j+1}}{(\Delta x)^{2}}+\tau \frac{u_{i}^{(n+1) j+1}-U_{i}^{j}}{\Delta t} \\
& =\frac{1}{\Delta x}\left[-\hat{p}\left(\frac{v_{i+1 / 2}^{(n)}-V_{i+1 / 2}^{+1}}{\Delta t}, x_{i+1 / 2}, t_{j+1 / 2}\right)-\hat{\beta}_{i+1 / 2}^{j+1 / 2}+\tau \frac{v_{i+1 / 2}^{(n) j+1}-V_{i+1 / 2}^{j}}{\Delta t}\right. \\
& \left.\quad+\hat{p}\left(\frac{v_{i-1 / 2}^{(n) j+1}-V_{i-1 / 2}^{j}}{\Delta t}, x_{i-1 / 2}, t_{j+1 / 2}\right)+\hat{\beta}_{i-1 / 2}^{j+1 / 2}-\tau \frac{v_{i-1 / 2}^{(n) j+1}-V_{i-1 / 2}^{j}}{\Delta t}\right] \\
& +\frac{\tau}{\Delta t}\left[\hat{\alpha}_{i}^{j+1}-\hat{\alpha}_{i}^{\prime}\right], \quad i=1, \ldots, I-1, \\
& \quad u_{0}^{(n+1) j+1}=u_{I}^{(n+1) j+1}=0 .
\end{aligned}
$$

For each given $j$, one repeatedly solves system (5.4) for the $v_{t-1 / 2}^{(n) j+1}$ and system (5.5) for the $u_{i}^{(n+1) j+1}$ starting from the initial approximations $v_{i-1 / 2}^{(0) j+1}=V_{i-1 / 2}^{j}$ or $u_{i}^{(0) \jmath+1}=U_{i}^{j}$ (on the first time step, the initial conditions (3.11c) and (3.13b) are used in the forms $V_{i-1 / 2}^{0}=\hat{g}\left(x_{i-1 / 2}\right)$ and $\left.U_{i}^{0}=g\left(x_{i}\right)\right)$ and accepts $u_{i}^{(n+1) j+1}$ as $U_{i}^{j+1}$ for the first $n$ such that inequality (5.3) is fulfilled. One then proceeds to repeat the process again on the next time step. In this particular implementation, 


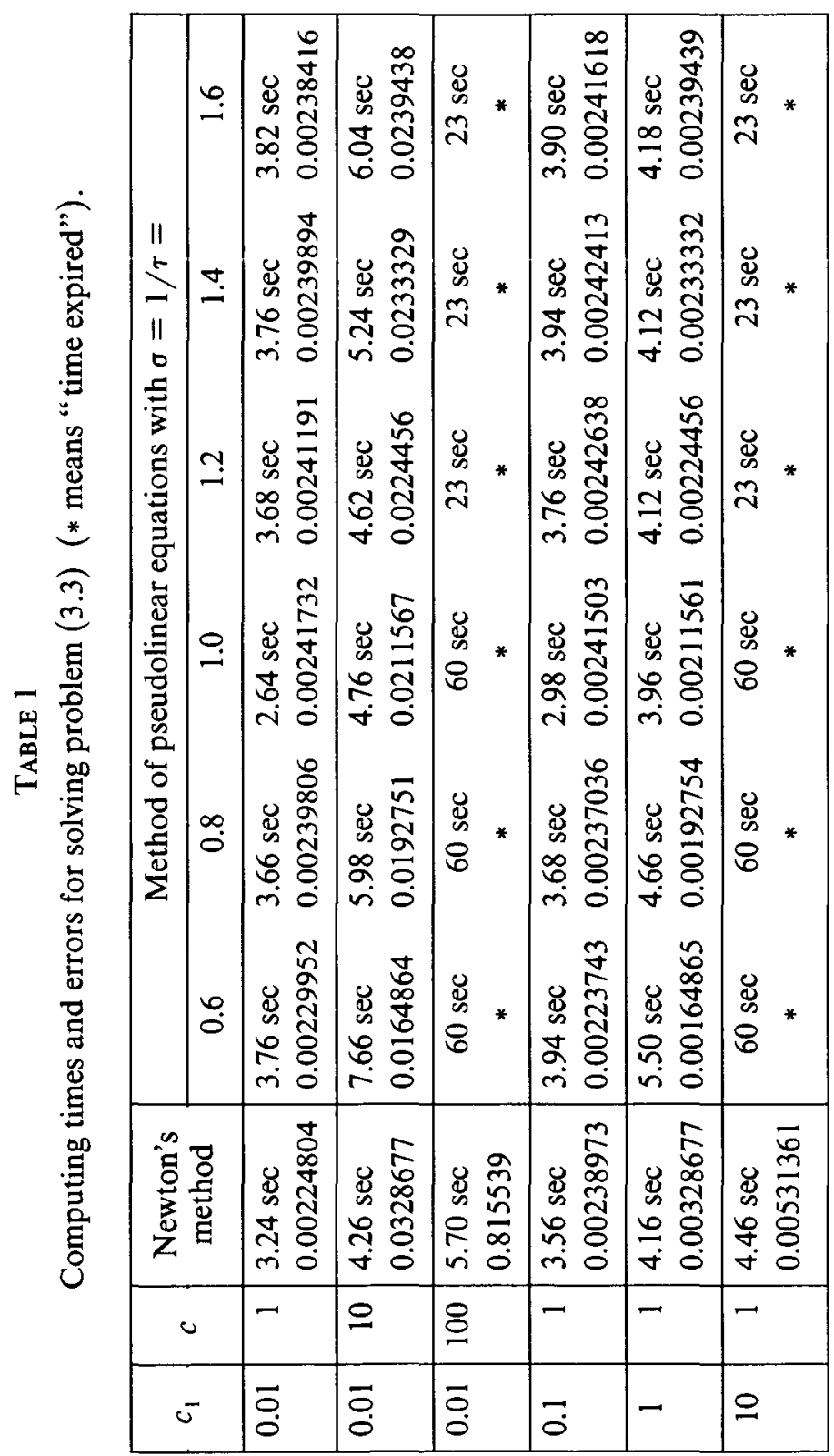




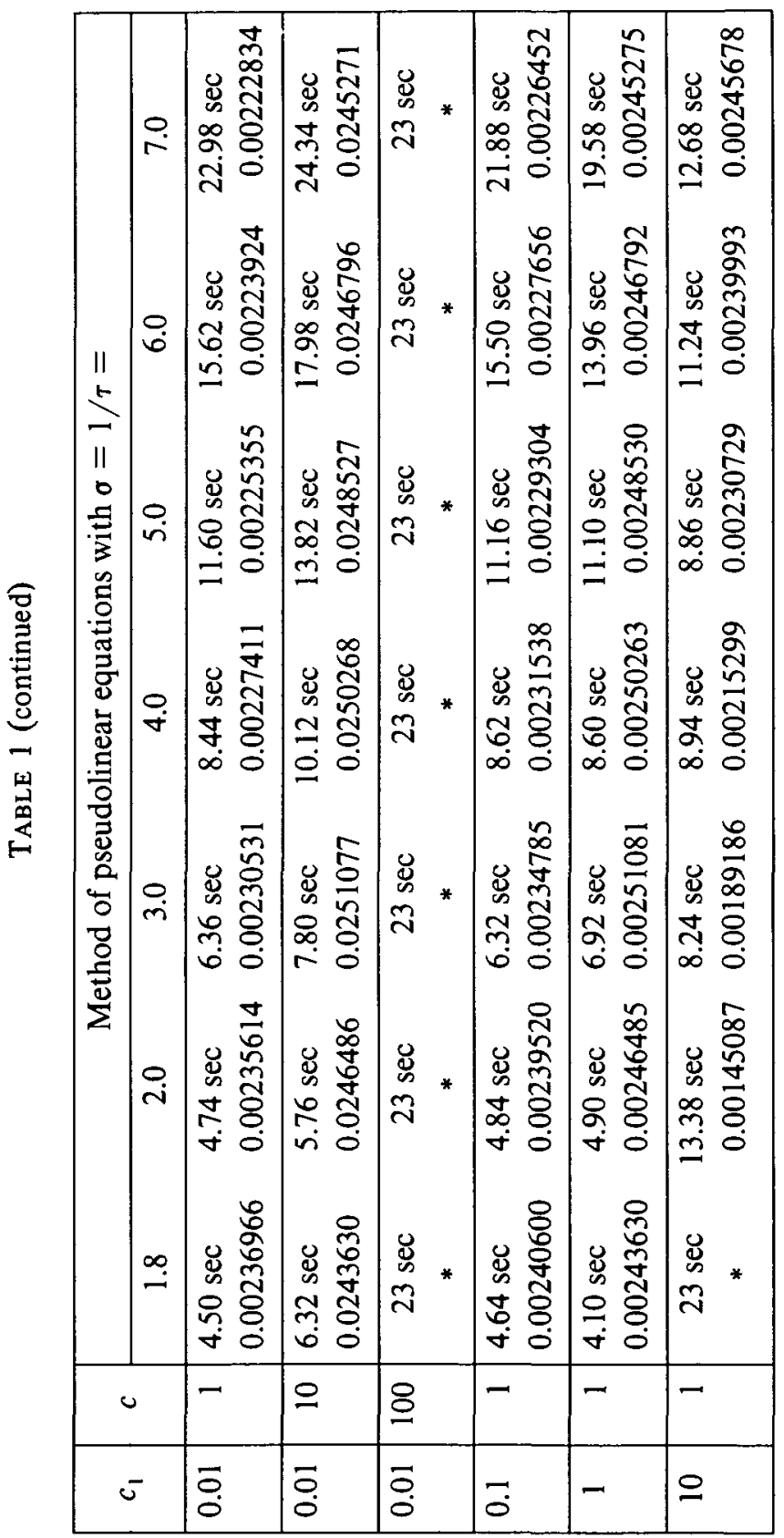


the method of Section 3 is used independently on each subdomain $(a, b) \times$ $\left(t_{j}, t_{j+1}\right)$ rather than on all of $D$ simultaneously. The value of the inverse function $\hat{p}(Y, x, t)$ is calculated by the standard Newton's method for scalar-valued functions. The $(k+1)$-st Newton iterate $X_{k+1}$ is accepted as $\hat{p}(Y, x, t)$ for the first $k$ such that

$$
\left|X_{k+1}-X_{k}\right| \leqslant \varepsilon_{2} \text {. }
$$

Computer programs for carrying out each of the three methods described above were coded and examples with the data

$$
\begin{gathered}
a=0, \quad b=1, \quad T=1, \quad p(X, x, t)=\left(1+c_{1} X^{2}\right) X, \quad g(x)=\hat{g}(x) \equiv 0, \\
f(x, t)=2 c t^{2}\left\{1+3 c_{1}\left[c t^{2}(1-2 x)\right]^{2}\right\}+2 c t x(1-x), \quad \beta(x, t) \equiv 0 \\
\alpha(x, t)=\int_{.5}^{x} f(\xi, t) d \xi, \quad \Delta x=0.1, \quad \Delta t=0.01, \quad \varepsilon_{1}=10^{-6}, \quad \varepsilon_{2}=10^{-8}
\end{gathered}
$$

(exact solution is $U=c t^{2} x(1-x)$ ) were run for various constants $c_{1}$ and $c$ on a BESM-6 at the Computing Center of the Siberian Branch of the Academy of Sciences of the USSR in Novosibirsk, the use of which the author acknowledges with gratitude. The symmetric tridiagonal matrix systems that arise during the computations were solved using Cholesky decomposition by the procedures of Martin and Wilkinson [6].

Numerical results for the two methods, designated "Newton's method" and "method of pseudolinear equations," are presented in Table 1. In each box of the table, two items are given: the first is the computing time (exclusive of compilation time and time for printing) quantified in units of $0.02 \mathrm{sec}$. and the second is a measure of the error at $t=T=J \Delta t$ of the approximate numerical solution versus the exact theoretical solution $U=c t^{2} x(1-x)$ given by

$$
\max _{1<i<I-1}\left|U_{1}^{J}-U\left(x_{1}, T\right)\right| \text {. }
$$

If the computations were not completed up to $t=T$, the computing time and the symbol * (denoting "time expired") were entered in the box.

From the data in Table 1, one sees that, for the two cases of "small nonlinearity," $c_{1}=0.01,0.1, c=1$, the method of pseudolinear equations with optimum parameters $\sigma=1, \tau=1$ (optimum with respect to computing time by comparison with the other entries for these cases) is $18.5 \%$ and $16.3 \%$ faster than Newton's method. However, for larger nonlinearities, the performance of the method of pseudolinear equations is not as good. For $c_{1}=1, c=1$ with optimum parameters $\sigma=1, \tau=1$, it is only $4.8 \%$ faster than Newton's method. For $c_{1}=0.01$, $c=10$ with optimum parameters $\sigma=1.2, \tau=1 / 1.2$, it is $8.5 \%$ slower. For $c_{1}=10, c=1$ with optimum parameters $\sigma=3, \tau=1 / 3$, it is $84.8 \%$ slower. Finally, for $c_{1}=0.01, c=100$, it fails to converge at all. 
A comparison of the optimum $\sigma$ observed in Table 1 with the minima and maxima over $\bar{D}$ of $\partial p\left(U_{x}, x, t\right) / \partial\left(U_{x}\right)$ is given in Table 2 . It shows that the optimum $\sigma$ lie between the minima and maxima, closer to the minima (cf. discussion of how to choose $\sigma$ in Section 3).

\section{TABLE 2}

Comparison of observed optimum $\sigma$ to minima and maxima of $\partial p\left(U_{x}, x, t\right) / \partial\left(U_{x}\right)$, with $U=c t^{2} x(1-x)$.

\begin{tabular}{|l|r|c|c|c|}
\hline$c_{1}$ & $c$ & $\min _{\bar{D}} \frac{\partial p\left(U_{x}, x, t\right)}{\partial\left(U_{x}\right)}$ & $\max _{\bar{D}} \frac{\partial p\left(U_{x}, x, t\right)}{\partial\left(U_{x}\right)}$ & $\begin{array}{c}\text { observed optimum } \\
\text { ofrom Table 1 }\end{array}$ \\
\hline 0.01 & 1 & 1 & 1.03 & 1 \\
0.01 & 10 & 1 & 4 & 1.2 \\
0.01 & 100 & 1 & 301 & - \\
0.1 & 1 & 1 & 1.3 & 1 \\
1 & 1 & 1 & 4 & 3 \\
10 & 1 & 1 & 31 & 1 \\
\hline
\end{tabular}

From the viewpoint of accuracy, the relative performance of the method of pseudolinear equations improves as the nonlinearity increases-just the opposite of the situation for computing time. For small nonlinearities $\left(c_{1}=0.01,0.1\right.$, $c=1$ ), the minimum and maximum of the relative errors for the method of pseudolinear equations are $0.89 \%\left(=0.00222834 / 0.25\right.$ for $c_{1}=0.01, c=1, \sigma=$ $7.0)$ and $0.97 \%\left(=0.00242638 / 0.25\right.$ for $\left.c_{1}=0.1, c=1, \sigma=1.2\right)$. The relative errors of Newton's method for these cases lie between these two values. For larger nonlinearities $\left(c_{1}=0.01, c=10\right.$ and $\left.c_{1}=1,10, c=1\right)$, however, the method of pseudolinear equations, which has relative errors between $0.58 \% \quad(=$ $0.00145087 / 0.25$ for $\left.c_{1}=10, c=1, \sigma=2.0\right)$ and $1.00 \%(=0.00251081 / 0.25$ for $c_{1}=1, c=1, \sigma=3.0$ ), is significantly more accurate than Newton's method, which has relative errors between $1.31 \%\left(=0.0328677 / 2.5\right.$ for $\left.c_{1}=0.01, c=10\right)$ and $2.13 \%\left(=0.00531361 / 0.25\right.$ for $\left.c_{1}=10, c=1\right)$.

\section{Concluding remarks}

The main computational advantage of the method of pseudolinear equations is that, on all iterations, only the right-hand side of the linear algebraic system to be solved need be recalculated. For Newton's method, on the other hand, both the right-hand side and the matrix must be recalculated on each iteration. 
In terms of overall applicability, the method of pseudolinear equations has one final advantage over Newton's method. The function $p$ is required by the method of pseudolinear equations to be only (boundedly) differentiable with respect to $X$ (see inequalities (2.3) and (2.4)), while Newton's method (Kantorovich and Akilov [3, Chapter 18]) requires roughly one additional order of differentiability.

\section{References}

[1] G. Geymonat and M. Sibony, "Approximation de certaines équations parabolıques non linéaires", Calcolo 13 (1976), 213-256.

[2] Thomas J. R. Hughes, "Unconditionally stable algorithms for nonlinear heat conduction", Comput. Methods Appl. Mech. Engrg. 10 (1977), 135-139.

[3] L. V. Kantorovich and G. P. Akilov, Functional analysis in normed spaces (Macmillan, New York, 1964).

[4] O. A. Ladyzhenskaya, Boundary-value problems of mathematical physics (in Russian) (Nauka, Moscow, 1973).

[5] John E. Lavery, "Solution of inhomogeneous quasilinear Dirichlet and Neumann problems by reduction to the Poisson equation and a posteriori error bounds", J. Reine Angew. Math. 299/300 (1978), 73-79.

[6] R. S. Martin and J. H. Wilkinson, "Symmetric decomposition of positive definite band matrices", Numer. Math. 7 (1965), 355-361.

[7] Robert D. Richtmyer and K. W. Morton, Difference methods for intial-value problems (Interscience, New York, 1967).

[8] A. A. Samarskĩ,, The theory of difference schemes (in Russian) (Nauka, Moscow, 1977). 